\title{
Effect of Nursing Care Standard on Postoperative Outcomes and Length of Hospital Stay in Children with Inguinal Hernia
}

\author{
Shadia Abd Elmoniem Syan, Hekmat Ibrahim Abed El-Kreem, Ibrahim Ali Ibrahim \& Eman Abd El-Aziz \\ Mohammed. \\ 1- Assistant lecturer of Pediatric Nursing, Faculty of Nursing, South Valley University, Qena, Egypt. \\ 2- Professor of Pediatric Nursing, Faculty of Nursing Assiut University, Egypt. \\ 3- Professor of Pediatric Surgery, Faculty of Medicine, Assuit University, Egypt. \\ 4- lecturer of Pediatric Nursing, Faculty of Nursing, South Valley University, Qena, Egypt.
}

Abstract

Inguinal hernia is a common condition requiring surgical repair within the pediatric group and it's one of the foremost usually performed surgical procedures within the world. The incidence of region hernias is threedimensional to five in term infants and thirteen in pre-term infants. Standard give direction and provide guidelines for performance of nursing staff and increase effectiveness of care. This study aimed to assess the effect of nursing care standard on postoperative outcomes and length of hospital stay in children with inguinal hernia. Methods: A quasi experimental research design conducted among one hundred twenty patients at Pediatric Surgery Unit Assiut University Children Hospital. Children were allocated to either study or control group. Three tools were used, Tool one: Structured interview sheet, tool two: designed standard of care and tool three: Evaluation sheet. Findings: Postoperative complications were highly significant in the control group than the study group. Conclusion: the standard of nursing care improving the postoperative outcomes for children with inguinal hernia as well as it reducing the length time of hospitalization. Recommendations: Considering the key role of nurses, the researchers hope that the results of this study can be implemented.

\section{Key words: Inguinal Hernia; Nursing Care Standard \& Children.}

\section{Introduction}

Inguinal hernia may be a common condition requiring surgical repair within the pediatric age bracket and it's one in every of the foremost unremarkably performed surgical procedures within the world. The incidence of region hernias is roughly three-dimensional to five in term infants and thirteen in pre-term infants. The incidence is highest during the first year of life with a peak during first month. (Alam et al., 2013) The processus vaginalis is a peritoneal sac that passes through the inguinal canal with the testis as it descends into the scrotum during the seventh to ninth month of gestation. In the last few weeks of gestation or shortly after birth, the layers of the processus vaginalis usually fuse along and obliterate the patency from the bodily cavity through the epithelial duct to the testes. When the processus vaginalis failed to close permits fluid or abdominal viscera to escape the peritoneal cavity and accounts for a variety of inguinal-scrotal abnormalities seen in infancy and childhood (Hughes \& Acerini, 2008).

Electively, hernia and hydrocele are diagnosed by a careful history and physical exam. A history of an intermittent bulge in the groin area, labia or scrotum should be confirmed by examining the child upright, while straining or crying, or by putting pressure on the abdomen. When no bulge is seen, palpable thickening of the spermatic cord (the silk glove sign) has over $90 \%$ sensitivity and specificity for presence of an inguinal hernia (Brandt, 2008).

Inguinal hernias are not known to resolve spontaneously and must therefore be repaired surgically shortly after diagnosis on an elective basis; the definitive treatment for inguinal hernia is early operation, a herniotomy. This will reduce the risks of incarceration with its attendant complications, such as obstruction and strangulation. For these complications to be avoided, timely diagnosis and operative technique are important (Abantanga \& Lakho, 2011).

Premature infants are more likely to develop incarcerated hernias, so most pediatric centers repair all premature infant hernias prior to discharge from hospital if the anesthetic risk is low. Otherwise the infant may return needing emergency surgery with a higher risk of surgical and anesthetic complications. Premature infants under $5 \mathrm{~kg}$ have a higher risk of postoperative testicular atrophy, hernia recurrence, cryptorchidism and hydrocele (Nagraj et al., 2006).

A standard is a model of established practice that is commonly accepted as correct. The care provided by nurses is guided by standard of care, which were developed and implemented to define the quality of care provided. Also standards give direction and provide guidelines for performance of nursing staff, increase effectiveness of care and improve efficiency, improve documentation of nursing care provided, 
determine the degree to which standards of nursing care maintained and take necessary corrective action in time, help supervisors to guide nursing staff to improve performance, improve basis for decisionmaking and devise alternative system for delivering nursing care (Marquis \& Huston, 2010).

Nurses need to understand the surgical procedure, its inherent risks, and knowledge of a patient's comorbidities to anticipate potential problems, perform focused assessments, and proactively manage the patient's care. Nurses use theoretical and evidencebased knowledge of human experiences and responses to collaborate with healthcare consumers to assess, diagnose, identify outcomes, plan, implement, and evaluate care. Nursing interventions are intended to produce beneficial effects, contribute to quality outcomes, and above all, do no harm. Nurses evaluate the effectiveness of their care in relation to identified outcomes and use evidence-based practice to improve care (Klipfel et al., 2010 \& American Nurses Association 2010).

\section{Significance of the study}

There is currently a tendency to shorten the length of hospital stay, with patients being discharged early to reduce medical costs and to increase the turnover rate of beds in the healthcare sector. Thus, healthcare professionals need to provide patients with the most effective and intensive care during relatively short stays in hospital. This has increased the introduction of standard of practice to assist clinical practitioners in their decision-making regarding the most appropriate healthcare for specific clinical circumstances (McKenzie et al., 2009).

\section{Aim of the study}

The aim of this study was to assess the effect of nursing care standard on postoperative outcomes and length of hospital stay in children with inguinal hernia.

\section{Hypothesis}

Children who receive nursing care standard have rapid wound healing process, decreased postoperative complications and decrease length of hospital stay than those children who not receive.

\section{Subjects \& Methods \\ Research design}

A quasi - experimental research design was utilized to conduct this study.

\section{Setting}

This study was conducted in the Pediatric Surgery Unit at Assiut University Children Hospital.

Pediatric Surgery Unit located at the fourth floor consisting from seven rooms; from these rooms there is one room for isolation of cases with specific conditions. Health care team includes five professors of pediatric surgery, three assistant lecturers, 2 nursing specialists and 12 nurses had technical nursing education.

\section{Subjects}

A convenience sample of 120 children suffering from inguinal hernia were selected from the previous setting and randomly divided into two groups of children (60 child for each group).the control group (60 child) were selected firstly, during sample collection the children given a number from one to five where number $1,3,5$ were selected and number 2,4 not taken in sample, after that the study group (60 child) were selected by the same technique as control group.

Two groups were distributed as follows:

Group I for control group.

Group II for study group.

Tools of information assortment

Tool (1): "Assessment of the characteristics of children and their clinical data" it includes two parts:

Part one: Characteristics of children include children's data (age, sex, birth order and residence)

Part two: Clinical data of children such as (type and site of inguinal hernia, date of admission, date of discharge, wound characteristics, etc.).

Tool (2): "Assessment of standard of nursing care observational checklist" It was developed by the researcher after reviewing of the related literatures and included six categories: admission care, preoperative care for the day before the operation, preoperative care for the operation day, immediate postoperative assessment, postoperative care and discharge plan, its score system (done $=2$, not done $=1$ for each category) (Piresetal., 2013 \& Park, 2010).

Tool (3): "Assessment of children's postoperative outcomes interview schedule" it was developed by the researcher after reviewing of the related literatures to assess the children's post -operative outcomes such as wound characteristics, superficial wound infection, urinary retention and recurrence and length of hospital stay.

\section{Method of data collection}

1- Official permission was obtained from the director of the Pediatric Surgery Unit.

2- Validity of three tools was calculated by five specialists in pediatric Field and its result was $95 \%$.

3- Reliability was estimated for three tool and it's result was $\mathrm{R}=0.68$ for tool one and two and $\mathrm{R}=$ 0.64 for tool three.

4- Assessment of socio - demographic characteristics of children was done by the researcher through using tool one. 
5- Developing of standard of care for inguinal hernia postoperatively: containing the following content: admission care, preoperative care for the day before the operation, preoperative care for the operation day, immediate postoperative assessment, postoperative care and discharge care.

6- A pilot study was carried out on 10 percent (12) of children who fulfilled the criteria of the study to test the feasibility and applicability of the tools and to make the needed correction. The children within the pilot study were excluded from study.

7- Steps of nursing care standard application on children include:

1- Developing nursing care standard, i.e planning\& determine its content.

2- Explain the content and method of application of nursing care standard for nurses.

3- Evaluate the performance of nurses who applied the standard on children through observational checklist.

4- Comparing the actual performance with the predetermined one.

5- Evaluate the deviation from the set standard.

6- Corrective action, if the performance meets the standard so, no correction method. When there is

Results

Table (1): Proportion distribution of the studied children regarding to their socio-demographic characteristics.

\begin{tabular}{|c|c|c|c|c|c|}
\hline \multirow{3}{*}{ Item } & \multicolumn{4}{|c|}{ Group } & \multirow{3}{*}{ P. value } \\
\hline & \multicolumn{2}{|c|}{ Study $n=60$} & \multicolumn{2}{|c|}{ Control $n=60$} & \\
\hline & No. & $\%$ & No. & $\%$ & \\
\hline \multicolumn{6}{|l|}{ Age } \\
\hline Birth up to $\downarrow 1$ year & 26 & 43.3 & 21 & 35.0 & \multirow{5}{*}{0.246} \\
\hline 1 year $-\downarrow 3$ year & 22 & 36.7 & 17 & 28.3 & \\
\hline 3 year $-\downarrow 6$ year & 6 & 10.0 & 12 & 20.0 & \\
\hline 6 year $-\downarrow 12$ year & 4 & 6.7 & 9 & 15.0 & \\
\hline 12 year and more & 2 & 3.3 & 1 & 1.7 & \\
\hline Mean \pm SD & \multicolumn{2}{|c|}{$30.8 \pm 38.2$} & \multicolumn{2}{|c|}{$39.2 \pm 42.8$} & 0.121 \\
\hline \multicolumn{6}{|l|}{ Sex } \\
\hline Male & 51 & 85.0 & 59 & 98.3 & \multirow{2}{*}{$0.008 * *$} \\
\hline Female & 9 & 15.0 & 1 & 1.7 & \\
\hline \multicolumn{6}{|l|}{ Gestational age/m } \\
\hline Mean \pm SD & \multicolumn{2}{|c|}{$8.6 \pm 0.6$} & \multicolumn{2}{|c|}{$8.8 \pm 0.4$} & $0.000 * *$ \\
\hline \multicolumn{6}{|l|}{ Birth weight/g } \\
\hline Mean \pm SD & \multicolumn{2}{|c|}{$2830 \pm 513$} & \multicolumn{2}{|c|}{$2821.7 \pm 764.3$} & $0.003 * *$ \\
\hline
\end{tabular}

Table (2): Proportion distribution of studied children regarding to their clinical data of the illness.

\begin{tabular}{|c|c|c|c|c|c|}
\hline \multirow{3}{*}{ Item } & \multicolumn{4}{|c|}{ Group } & \multirow{3}{*}{ P. value } \\
\hline & \multicolumn{2}{|c|}{ Study $n=60$} & \multicolumn{2}{|c|}{ Control $n=60$} & \\
\hline & No. & $\%$ & No. & $\%$ & \\
\hline \multicolumn{6}{|l|}{ Type of inguinal hernia } \\
\hline Non complicated inguinal hernia & 35 & 58.3 & 42 & 70.0 & \multirow{2}{*}{0.396} \\
\hline Complicated inguinal hernia & 19 & 31.7 & 13 & 21.7 & \\
\hline
\end{tabular}

deviation from standard, so the correction of performance is needed.

-The scoring system: done item was taken two and not done item was taken one.

8- The children in cluster one (control group) followed the routine medical care.

9- Assessment of children postoperative outcomes by applying tool three at the third day of inguinal hernia surgery.

10 - Data were collected throughout period from the first of Dec 2015 to the end of August 2017; each questionnaire was filled throughout 15 minutes.

\section{Statistical analysis of data}

Data were collected, tabulated and analyzed. Data entry was done by (Microsoft stand out 2007 computer soft- ware package).whereas applied mathematics analysis was done by using SPSS sixteen. Applied mathematics software system package and stand out for figures. Using descriptive statistics within the kind of frequencies and percentages for qualitative variables and mean and standard deviations for quantitative variables. Qualitative variables were compared using Chisquare. Statistical significance was considered at Pvalue $<0.05$ 


\begin{tabular}{|c|c|c|c|c|c|}
\hline \multirow{3}{*}{ Item } & \multicolumn{4}{|c|}{ Group } & \multirow{4}{*}{ P. value } \\
\hline & \multicolumn{2}{|c|}{ Study $n=60$} & \multicolumn{2}{|c|}{ Control $n=60$} & \\
\hline & No. & $\%$ & No. & $\%$ & \\
\hline Recurrent inguinal hernia & 6 & 10.0 & 5 & 8.3 & \\
\hline \multicolumn{6}{|l|}{ Site of inguinal hernia } \\
\hline Right side & 42 & 70.0 & 29 & 48.3 & \multirow{3}{*}{$0.042 *$} \\
\hline Left side & 15 & 25.0 & 23 & 38.3 & \\
\hline Bilateral & 3 & 5.0 & 8 & 13.3 & \\
\hline
\end{tabular}

Table (3): The comparison between the studied children regarding to preparation for receiving child in Pediatric Surgery Care Unit (PSCU).

\begin{tabular}{|c|c|c|c|c|c|c|c|c|c|}
\hline \multirow{4}{*}{ Item } & \multicolumn{8}{|c|}{ Group } & \multirow{4}{*}{ P. value } \\
\hline & \multirow{2}{*}{\multicolumn{2}{|c|}{$\begin{array}{l}\text { Study } \\
\text { Done }\end{array}$}} & \multicolumn{2}{|c|}{$\mathrm{n}=60$} & \multicolumn{4}{|c|}{ Control $n=60$} & \\
\hline & & & No & done & \multicolumn{2}{|c|}{ Done } & \multicolumn{2}{|c|}{ Not done } & \\
\hline & No. & $\%$ & No. & $\%$ & No. & $\%$ & No. & $\%$ & \\
\hline $\begin{array}{l}\text { 1. Receives prior } \\
\text { information from pediatric } \\
\text { emergency }\end{array}$ & 0 & 0.0 & 60 & 100.0 & 0 & 0.0 & 60 & 100.0 & NA \\
\hline $\begin{array}{l}\text { 2. Keeps articles for suction } \\
\text { ready }\end{array}$ & 46 & 76.7 & 14 & 23.3 & 0 & 0.0 & 60 & 100.0 & $0.000 * *$ \\
\hline $\begin{array}{l}\text { 3. Keeps ready an oxygen } \\
\text { source with adequate } \\
\text { distilled water }\end{array}$ & 55 & 91.7 & 5 & 8.3 & 13 & 21.7 & 47 & 78.3 & $0.000 * *$ \\
\hline 4. Weighs the child & 60 & 100.0 & 0 & 0.0 & 60 & 100.0 & 0 & 0.0 & NA \\
\hline $\begin{array}{l}\text { 5. Assesses child for color } \\
\text { and respiratory status }\end{array}$ & 58 & 96.7 & 2 & 3.3 & 43 & 71.7 & 17 & 28.3 & $0.000 * *$ \\
\hline $\begin{array}{l}\text { 6.Keeps child NPO } \\
\text { according to case }\end{array}$ & 60 & 100.0 & 0 & 0.0 & 60 & 100.0 & 0 & 0.0 & NA \\
\hline $\begin{array}{l}\text { 7. Physical assessment } \\
\text { (weight). }\end{array}$ & 60 & 100.0 & 0 & 0.0 & 60 & 100.0 & 0 & 0.0 & NA \\
\hline \multicolumn{10}{|l|}{ 8. Physiological assessment } \\
\hline 8.1 Temperature & 59 & 98.3 & 1 & 1.7 & 21 & 35.0 & 39 & 65.0 & $0.000^{* * *}$ \\
\hline 8.2 Respiration & 49 & 81.7 & 11 & 18.3 & 11 & 18.3 & 49 & 81.7 & $0.000 * *$ \\
\hline 8.3 B.P & 39 & 65.0 & 21 & 35.0 & 0 & 0.0 & 60 & 100.0 & $0.000 * *$ \\
\hline 8.4 Pulse. & 41 & 68.3 & 19 & 31.7 & 3 & 5.0 & 57 & 95.0 & $0.000 * *$ \\
\hline
\end{tabular}

Table (4): The relationship between the studied children regarding to nursing care of intravenous cannulas.

\begin{tabular}{|c|c|c|c|c|c|c|c|c|c|}
\hline \multirow{4}{*}{ Item } & \multicolumn{8}{|c|}{ Group } & \multirow{4}{*}{ P. value } \\
\hline & \multicolumn{4}{|c|}{ Study $\quad n=60$} & \multicolumn{4}{|c|}{ Control $n=60$} & \\
\hline & \multicolumn{2}{|c|}{ Done } & \multicolumn{2}{|c|}{ Not done } & \multicolumn{2}{|c|}{ Done } & \multicolumn{2}{|c|}{ Not done } & \\
\hline & No. & $\%$ & No. & $\%$ & No. & $\%$ & No. & $\%$ & \\
\hline 1. Wash hands & 60 & 100.0 & 0 & 0.0 & 60 & 100.0 & 0 & 0.0 & NA \\
\hline $\begin{array}{l}\text { 2. Organize equipment on clean } \\
\text { area in order of use. }\end{array}$ & 51 & 85.0 & 9 & 15.0 & 21 & 35.0 & 39 & 65.0 & $0.000 * *$ \\
\hline $\begin{array}{l}\text { 3. Open sterile packages using } \\
\text { sterile technique. }\end{array}$ & 54 & 90.0 & 6 & 10.0 & 21 & 35.0 & 39 & 65.0 & $0.000 * *$ \\
\hline 4. Wear disposable gloves. & 30 & 50.0 & 30 & 50.0 & 14 & 23.3 & 46 & 76.7 & $0.002 * *$ \\
\hline \multicolumn{10}{|l|}{ 5. Cleans insertion site } \\
\hline 5.1 Using antiseptic solution & 60 & 100.0 & 0 & 0.0 & 3 & 5.0 & 57 & 95.0 & $0.000 * *$ \\
\hline 5.2 Using circular motion & 51 & 85.0 & 9 & 15.0 & 0 & 0.0 & 60 & 100.0 & $0.000 * *$ \\
\hline 6. Quickly connect needle adapter & 60 & 100.0 & 0 & 0.0 & 60 & 100.0 & 0 & 0.0 & NA \\
\hline
\end{tabular}




\begin{tabular}{|c|c|c|c|c|c|c|c|c|c|}
\hline \multirow{4}{*}{ Item } & \multicolumn{8}{|c|}{ Group } & \multirow{4}{*}{ P. value } \\
\hline & \multicolumn{4}{|c|}{ Study $n=60$} & \multicolumn{4}{|c|}{ Control $n=60$} & \\
\hline & \multicolumn{2}{|c|}{ Done } & \multicolumn{2}{|c|}{ Not done } & \multicolumn{2}{|c|}{ Done } & \multicolumn{2}{|c|}{ Not done } & \\
\hline & No. & $\%$ & No. & $\%$ & No. & $\%$ & No. & $\%$ & \\
\hline $\begin{array}{l}\text { of administration set to hub of } \\
\text { butterfly tubing \&don't touch } \\
\text { point of entry needle adapter. }\end{array}$ & & & & & & & & & \\
\hline 7. Apply sterile dressing over site. & 0 & 0.0 & 60 & 100.0 & 0 & 0.0 & 60 & 100.0 & NA \\
\hline $\begin{array}{l}\text { 8. Dispose of used needles in } \\
\text { appropriate sharps container. }\end{array}$ & 60 & 100.0 & 0 & 0.0 & 60 & 100.0 & 0 & 0.0 & NA \\
\hline 9. Discard supplies & 60 & 100.0 & 0 & 0.0 & 53 & 88.3 & 7 & 11.7 & $0.006 * *$ \\
\hline 10. Remove gloves & 30 & 50.0 & 30 & 50.0 & 43 & 71.7 & 17 & 28.3 & $0.015^{*}$ \\
\hline $\begin{array}{l}\text { 11. Observe insertion site for } \\
\text { absence of infiltration, phlebitis, } \\
\text { or inflammation. }\end{array}$ & 60 & 100.0 & 0 & 0.0 & 39 & 65.0 & 21 & 35.0 & $0.000 * *$ \\
\hline 12. Wash hands. & 60 & 100.0 & 0 & 0.0 & 50 & 83.3 & 10 & 16.7 & $0.001 * *$ \\
\hline
\end{tabular}

Table (5):The relationship between the studied children relating to the nursing assessment in immediate postoperative interval (first 2 hour).

\begin{tabular}{|c|c|c|c|c|c|c|c|c|c|}
\hline \multirow{4}{*}{ Item } & \multicolumn{8}{|c|}{ Group } & \multirow{4}{*}{ P. value } \\
\hline & \multicolumn{4}{|c|}{ Study $n=60$} & \multicolumn{4}{|c|}{ Control $n=60$} & \\
\hline & \multicolumn{2}{|c|}{ Done } & \multicolumn{2}{|c|}{ Not done } & \multicolumn{2}{|c|}{ Done } & \multicolumn{2}{|c|}{ Not done } & \\
\hline & No. & $\%$ & No. & $\%$ & No. & $\%$ & No. & $\%$ & \\
\hline $\begin{array}{l}\text { 1. Record time of child arrival } \\
\text { at the nursing unit. }\end{array}$ & 0 & 0.0 & 60 & 100.0 & 39 & 65.0 & 21 & 35.0 & $0.000 * *$ \\
\hline 2. Place child in safe position. & 60 & 100.0 & 0 & 0.0 & 10 & 16.70 & 50 & 83.30 & $0.000 * *$ \\
\hline $\begin{array}{l}\text { 3. Note the level of child } \\
\text { consciousness }\end{array}$ & 57 & 95.0 & 3 & 5.0 & 0 & 0.0 & 60 & 100.0 & $0.000 * *$ \\
\hline \multicolumn{10}{|l|}{$\begin{array}{l}\text { 4. Record vital signs correctly } \\
\text { and regularly. }\end{array}$} \\
\hline $\begin{array}{l}4.1 \text { Every } 15 \text { minutes the first } \\
\text { hour. }\end{array}$ & 0 & 0.0 & 60 & 100.0 & 0 & 0.0 & 60 & 100.0 & NA \\
\hline $\begin{array}{l}\text { 4.1. Every } 30 \text { minutes the } \\
\text { second hour. }\end{array}$ & 0 & 0.0 & 60 & 100.0 & 0 & 0.0 & 60 & 100.0 & NA \\
\hline 5. Provide for warmth. & 60 & 100.0 & 0 & 0.0 & 60 & 100.0 & 0 & 0.0 & $\mathrm{NA}$ \\
\hline $\begin{array}{l}\text { 6. Assess skin color and } \\
\text { condition }\end{array}$ & 60 & 100.0 & 0 & 0.0 & 39 & 65.0 & 21 & 35.0 & $0.000 * *$ \\
\hline $\begin{array}{l}\text { 7. Observe dressing for } \\
\text { color.\& odor. }\end{array}$ & 60 & 100.0 & 0 & 0.0 & 52 & 86.70 & 8 & 13.30 & $0.003 * *$ \\
\hline 8. Check dressing for bleeding & 60 & 100.0 & 0 & 0.0 & 52 & 86.70 & 8 & 13.30 & $0.003 * *$ \\
\hline \multicolumn{10}{|l|}{ 9. Record I.V fluid. } \\
\hline $\begin{array}{l}9.1 \text { Observe rate of fluid } \\
\text { intake. }\end{array}$ & 60 & 100.0 & 0 & 0.0 & 29 & 48.30 & 31 & 51.70 & $0.000 * *$ \\
\hline 9.2 Observe site of cannula. & 60 & 100.0 & 0 & 0.0 & 39 & 65.0 & 21 & 35.0 & $0.000 * *$ \\
\hline 9.3 Type of fluid. & 60 & 100.0 & 0 & 0.0 & 57 & 95.0 & 3 & 5.0 & 0.079 \\
\hline $\begin{array}{l}\text { 10. Evaluate pain and give } \\
\text { medication as order. }\end{array}$ & 60 & 100.0 & 0 & 0.0 & 0 & 0.0 & 60 & 100.0 & $0.000 * *$ \\
\hline
\end{tabular}


Table (6): The relationship between the studied children concerning to nursing management of surgical wound.

\begin{tabular}{|c|c|c|c|c|c|c|c|c|c|}
\hline \multirow{4}{*}{ Item } & \multicolumn{8}{|c|}{ Group } & \multirow{4}{*}{ P. value } \\
\hline & \multicolumn{4}{|c|}{ Study $n=60$} & \multicolumn{4}{|c|}{ Control $n=60$} & \\
\hline & \multicolumn{2}{|c|}{ Done } & \multicolumn{2}{|c|}{ Not done } & \multicolumn{2}{|c|}{ Done } & \multicolumn{2}{|c|}{ Not done } & \\
\hline & No. & $\%$ & No. & $\%$ & No. & $\%$ & No. & $\%$ & \\
\hline $\begin{array}{l}\text { 1. Prepare equipment } \& \\
\text { supplies }\end{array}$ & 60 & 100.0 & 0 & 0.0 & 42 & 70.0 & 18 & 30.0 & $0.000 * *$ \\
\hline $\begin{array}{l}\text { 2. Explain procedure to child's } \\
\text { parent }\end{array}$ & 0 & 0.0 & 60 & 100.0 & 7 & 11.70 & 53 & 88.30 & $0.006 * *$ \\
\hline 3.Wash hands & 60 & 100.0 & 0 & 0.0 & 60 & 100.0 & 0 & 0.0 & $\mathrm{NA}$ \\
\hline 4. Protects child's privacy & 55 & 91.70 & 5 & 8.30 & 39 & 65.0 & 21 & 35.00 & $0.000 * *$ \\
\hline $\begin{array}{l}\text { 5. Wear clean exam gloves \& } \\
\text { mask. }\end{array}$ & 60 & 100.0 & 0 & 0.0 & 41 & 68.30 & 19 & 31.70 & $0.000 * *$ \\
\hline $\begin{array}{l}\text { 6. Assess need for pain } \\
\text { medication }\end{array}$ & 41 & 68.30 & 19 & 31.70 & 2 & 3.30 & 58 & 96.70 & $0.000 * *$ \\
\hline 7. Remove old dressings & 60 & 100.0 & 0 & 0.0 & 60 & 100.0 & 0 & 0.0 & $\mathrm{NA}$ \\
\hline $\begin{array}{l}\text { 8. Checking wound for:.color, } \\
\text { cleanness etc }\end{array}$ & 60 & 100.0 & 0 & 0.0 & 60 & 100.0 & 0 & 0.0 & NA \\
\hline $\begin{array}{l}\text { 9. Remove and discard gloves. } \\
\text { Done a pair of clean gloves }\end{array}$ & 0 & 0.0 & 60 & 100.0 & 0 & 0.0 & 60 & 100.0 & NA \\
\hline \multicolumn{10}{|l|}{$\begin{array}{l}\text { 10. Clean wound with } \\
\text { antiseptic solution. }\end{array}$} \\
\hline $\begin{array}{l}\text { 10.1. Use one gauze for each } \\
\text { wipe, from least contaminated } \\
\text { to more contaminated area }\end{array}$ & 60 & 100.0 & 0 & 0.0 & 21 & 35.00 & 39 & 65.0 & $0.000 * *$ \\
\hline $\begin{array}{l}\text { 10.2. Dry wound using sponge } \\
\text { in same motion }\end{array}$ & 60 & 100.0 & 0 & 0.0 & 24 & 40.0 & 36 & 60.0 & $0.00 * *$ \\
\hline $\begin{array}{l}\text { 11.Apply prescribed antibiotic } \\
\text { ointments. }\end{array}$ & 60 & 100.0 & 0 & 0.0 & 59 & 98.30 & 1 & 1.70 & 0.315 \\
\hline $\begin{array}{l}\text { 12. Remove and discard } \\
\text { gloves. wear sterile gloves. }\end{array}$ & 0 & 0.0 & 60 & 100.0 & 0 & 0.0 & 60 & 100.0 & NA \\
\hline $\begin{array}{l}\text { 13. Apply clean dressing on } \\
\text { wound. }\end{array}$ & 60 & 100.0 & 0 & 0.0 & 59 & 98.30 & 1 & 1.70 & 0.315 \\
\hline $\begin{array}{l}\text { 14. Remove and discard } \\
\text { gloves. }\end{array}$ & 60 & 100.0 & 0 & 0.0 & 59 & 98.30 & 1 & 1.70 & 0.315 \\
\hline 15. Wash hands. & 60 & 100.0 & 0 & 0.0 & 60 & 100.0 & 0 & 0.0 & $\mathrm{NA}$ \\
\hline \multicolumn{10}{|l|}{ 16. Documentation and report. } \\
\hline $\begin{array}{l}\text { 16.1. Date and time of } \\
\text { dressing done. }\end{array}$ & 58 & 96.70 & 2 & 3.30 & 2 & 3.30 & 58 & 96.70 & $0.000 * *$ \\
\hline $\begin{array}{l}\text { 16.2. Brief description of the } \\
\text { wound site. }\end{array}$ & 55 & 91.70 & 5 & 8.30 & 0 & 0.0 & 60 & 100.0 & $0.000 * *$ \\
\hline $\begin{array}{l}\text { 16. 3. Brief description of the } \\
\text { site care done and dressing } \\
\text { applied. }\end{array}$ & 0 & 0.0 & 60 & 100.0 & 0 & 0.0 & 60 & 100.0 & NA \\
\hline
\end{tabular}


Table (7): The relationship between the studied children concerning to nursing management of nutrition.

\begin{tabular}{|c|c|c|c|c|c|c|c|c|c|}
\hline \multirow{4}{*}{ Item } & \multicolumn{8}{|c|}{ Group } & \multirow{4}{*}{ P. value } \\
\hline & \multicolumn{4}{|c|}{ Study $n=60$} & \multicolumn{4}{|c|}{ Control $n=60$} & \\
\hline & \multicolumn{2}{|c|}{ Done } & \multicolumn{2}{|c|}{\begin{tabular}{|l|} 
Not done \\
\end{tabular}} & \multicolumn{2}{|c|}{ Done } & \multicolumn{2}{|c|}{ Not done } & \\
\hline & No. & $\%$ & No. & $\%$ & No. & $\%$ & No. & $\%$ & \\
\hline $\begin{array}{l}\text { 1. Encourage breast feeding for } \\
\text { breastfed infants. }\end{array}$ & 60 & 100.0 & 0 & 0.0 & 60 & 100.0 & 0 & 0.0 & NA \\
\hline \multicolumn{10}{|l|}{ 2. older children up to 2 years: } \\
\hline $\begin{array}{l}\text { 2.1. Keep patient room free } \\
\text { from bad odor. }\end{array}$ & 60 & 100.0 & 0 & 0.0 & 42 & 70.0 & 18 & 30.0 & $0.000 * *$ \\
\hline $\begin{array}{l}\text { 2.2. Give mouth care before and } \\
\text { after meals. }\end{array}$ & 57 & 95.0 & 3 & 5.0 & 0 & 0.0 & 60 & 100.0 & $0.000 * *$ \\
\hline 2.3. Give ordered antiemetic & 60 & 100.0 & 0 & 0.0 & 49 & 81.70 & 11 & 18.30 & $0.001 * *$ \\
\hline 2.4. Give small frequent meals. & 55 & 91.70 & 5 & 8.30 & 11 & 18.30 & 49 & 81.70 & $0.000 * *$ \\
\hline $\begin{array}{l}2.5 . \text { Encourage adequate } \\
\text { calories in meals, vitamins, } \\
\text { carbohydrates and supplements } \\
\text { between meals. }\end{array}$ & 52 & 86.70 & 8 & 13.3 & 0 & 0.0 & 60 & 100.0 & $0.000 * *$ \\
\hline
\end{tabular}

Table (8): The relationship between the studied children concerning to their wound characteristics postoperatively.

\begin{tabular}{|c|c|c|c|c|c|}
\hline \multirow{3}{*}{ Item } & \multicolumn{4}{|c|}{ Group } & \multirow{3}{*}{ P. value } \\
\hline & \multicolumn{2}{|c|}{ Study $n=60$} & \multicolumn{2}{|c|}{ Control $n=60$} & \\
\hline & No. & $\%$ & No. & $\%$ & \\
\hline \multicolumn{6}{|l|}{ Type of surgical wound } \\
\hline Clean wound & 60 & 100.0 & 60 & 100.0 & NA \\
\hline \multicolumn{6}{|l|}{ Redness } \\
\hline No & 40 & 66.7 & 30 & 50.0 & \multirow{2}{*}{0.064} \\
\hline Yes & 20 & 33.3 & 30 & 50.0 & \\
\hline \multicolumn{6}{|l|}{ Swelling } \\
\hline No & 52 & 86.7 & 29 & 48.3 & \multirow{2}{*}{$0.000 * *$} \\
\hline Yes & 8 & 13.3 & 31 & 51.7 & \\
\hline \multicolumn{6}{|l|}{ Bleeding } \\
\hline No & 60 & 100.0 & 57 & 95.0 & \multirow{2}{*}{0.079} \\
\hline Yes & 0 & 0.0 & 3 & 5.0 & \\
\hline \multicolumn{6}{|l|}{ Presence of discharge } \\
\hline No & 60 & 100.0 & 47 & 78.3 & \multirow{2}{*}{$0.000 * *$} \\
\hline Yes & 0 & 0.0 & 13 & 21.7 & \\
\hline \multicolumn{6}{|l|}{ Type of discharge if yes $n=13$} \\
\hline Serogenouse & 0 & 0.0 & 9 & 69.2 & \multirow{3}{*}{$0.000 * *$} \\
\hline Pus & 0 & 0.0 & 2 & 15.4 & \\
\hline Blood & 0 & 0.0 & 2 & 15.4 & \\
\hline \multicolumn{6}{|l|}{ Length of hospital stay/days } \\
\hline Mean \pm SD & \multicolumn{2}{|c|}{$3.2 \pm 0.5$} & \multicolumn{2}{|c|}{$4.9 \pm 2.1$} & $0.000 * *$ \\
\hline
\end{tabular}


Table (9): The relationship between the studied children concerning to their post-operative complications.

\begin{tabular}{|c|c|c|c|c|c|}
\hline \multirow{3}{*}{ Item } & \multicolumn{4}{|c|}{ Group } & \multirow{3}{*}{ P. value } \\
\hline & \multicolumn{2}{|c|}{$\begin{array}{ll}\text { Study } & \mathrm{n}=60\end{array}$} & \multicolumn{2}{|c|}{ Control $\quad \mathrm{n}=\mathbf{6 0}$} & \\
\hline & No. & $\%$ & No. & $\%$ & \\
\hline \multicolumn{6}{|l|}{ Scrotal swelling } \\
\hline 3 days & 42 & 70.0 & 58 & 96.7 & $<0.001 * *$ \\
\hline Superficial wound infection & 0 & 0.0 & 0 & 0.0 & - \\
\hline \multicolumn{6}{|l|}{ Fever } \\
\hline 3 days & 8 & 13.3 & 29 & 48.3 & $<0.001 * *$ \\
\hline \multicolumn{6}{|l|}{ Urinary retention } \\
\hline 3 days & 5 & 8.3 & 12 & 20.0 & 0.067 \\
\hline
\end{tabular}

Table (1): Portrays proportion distribution of the studied children regarding to their socio-demographic characteristics. One third of subjects $(43.3 \%$ $\& 35 \%$ ) within the study and control groups severally were within the age group birth up to less than one year, whereas over one third of them within the study cluster thirty six.7\% and $28.3 \%$ of them within the control cluster were within the age bracket $1-\downarrow 3$ years. The mean gestational age was $8.6 \pm 0.6 \& 8.8 \pm 0.4$ month in the study and control clusters respectively.

Table (2): Presents proportion distribution of studied children regarding to their clinical data of the illness. Where two 2 thirds (70\%) and nearly (48.3\%) of children within the study and control clusters severally had right sided herniation.With solely $10 \%$ $\& 8.3 \%$ of children in both study and control clusters respectively had repeated herniation.

Table (3): Illustrate the comparison between the studied children regarding to preparation for receiving child in Pediatric Surgery Care Unit. A highly significant difference was found between study and control groups at the $\mathrm{P}$ Value of $0.000^{* *}$. Where keeping equipment for suction ready was done for $76.7 \%$ of children in study group compared to none in control group. Regarding physiological assessment it revealed that temperature assessment was done in the majority of children $(98.3 \%)$ in study group compared to thirty fifth percent of them in control cluster.

Table (4): Illustrate the relationship between the studied children regarding to nursing care of intravenous cannulas. A highly significant difference was found at the $\mathrm{P}$ Value of $0.000^{* *}$. Where open sterile packages using sterile technique was done in more than two thirds of children $(90 \%)$ in study group compared to one third (35\%) in control group. Using antiseptic solution was done for all children $(100 \%)$ in study group compared to only $5.0 \%$ of children in control group. While observe insertion site for absence of infiltration, phlebitis or inflammation was done for all children (100\%) in study group compared to nearly two thirds (65\%) of children in control group.

Table (5): Portrays the relationship between the studied children relating to the nursing assessment in immediate postoperative interval (first 2 hour). A highly significant difference was found at the $\mathrm{P}$ Value of $0.000^{* *}$. Where Record time of child arrival at the nursing unit was done for more than half of children $65 \%$ in control group compared to none in study group, While checking the level of child consciousness was done for $95 \%$ of children in study group compared to none in control group. Also the observing of dressing for color\& odor and checking the dressing for bleeding were done for all children $(100 \%)$ in study group compared to $86.70 \%$ in control group at the P Value of $0.003^{* *}$.

Table (6): Illustrates the relationship between the studied children concerning to nursing management of surgical wound. A highly significant difference was found at the $\mathrm{P}$ Value of $0.000^{* *}$.Preparing equipment and supplies that were done for all children $100 \%$ in study group compared to more than two thirds of children $70 \%$ in control group. Wearing a clean exam gloves and mask were done for all children $100 \%$ in study group compared to two thirds of children $68.30 \%$ in control group. Use one gauze for each wipe, from least contaminated to more contaminated area was done for all children $100 \%$ in study group compared to one third $35 \%$ of children in control group. Brief description of the wound site was done for $91.70 \%$ of children in study group compared to none in control group.

Table (7) Shows the relationship between the studied children concerning to nursing management of nutrition. A highly significant difference was found at the $\mathrm{P}$ Value of $0.000^{* *}$. Where mouth care was done before and after meals for $95 \%$ of children in study group compared to none in control group. Also encourage adequate calories in meals, vitamins, carbohydrates and supplements between meals was done for $86.70 \%$ of children in study group compared to none in control group. 
Table (8): Presents the relationship between the studied children regarding to their wound characteristics postoperatively. There was high significant difference as the $\mathrm{P}$ Values were equal $<0.001 * *$ where only $13.3 \%$ of children in study group compared to more than half of children $51.7 \%$ in control group had swelling wound. There was nearly fourth $21.7 \%$ of children in control group had wound discharge compared to none in study group, where $69.2 \%$ of children in control group had Serogenouse discharge and $15.4 \%$ of children had pus and blood discharge.

Table (9): Presents the relationship between the studied children regarding to their post-operative complications. There was high significant difference as the $\mathrm{P}$ Values were equal $<0.001 * *$ where in the third day of operation there was more than two thirds of children $70 \%$ in study group who had scrotal swelling compared to the majority of children $96.7 \%$ in control group. Regarding to Fever: it was revealed that nearly half of children $(48.3 \%)$ in control group had fever in the $3^{\text {rd }}$ day of surgery compared to only $13.3 \%$ of children in study group. Regarding to the Recurrence of inguinal hernia :there is no recurrent cases of inguinal hernia in both groups.

\section{Discussion}

Health-care professionals frequently request systematic evidence-based guidance to facilitate decision-making in the provision of clinical care. They also need adequate support from hospital management to lead changes in practice. One approach to the provision of such guidance is the use of standards of care as an implementation tool. They are often developed by translating guidelines into local protocols for application in clinical practice. Although standards of care are being used worldwide, evidence about their usefulness has been unclear (Rotter etal., 2010 \&WHO, 2004).

Indirect inguinal hernias can occur at all ages, with highest incidence during the first year of life and its peak during first month (Alametal., 2013). The results of this study revealed that more than one third of children with congenital inguinal hernia $(43.3 \% \& 35 \%)$ in each study and control clusters respectively were within the age group from birth up to one year. additionally solely $3.3 \%$ of children in the study cluster and $1.7 \%$ of them in control cluster were at the age group 12years and more (table 1), this was consistent with the study of Osifo \& Osaigbovo (2008) who reported that the prevalence of Patent Processus Vaginalis (PPV) is highest throughout infancy and declines with age.

The relationship between the processus vaginalis with testicular descent explained why more than $90 \%$ of inguinal hernias in pediatric are diagnosed in boys
(Stevenetal, 2011). The results of this study revealed that the majority of inguinal hernia cases were male $(85 \%$ \&98.3\%) respectively in study and control clusters (table 1), this was consistent with Kareem \& Juma'a (2009) who conducted a study on 252 patients with inguinal hernia were collected in Baquba general hospital-Diayla-Iraq . From the total number, 8 cases only were female and the remaining 244 cases were male.

The right-sided preponderance is related to the later descent of the right testicle and later obliteration of the processus vaginalis (Brandt, 2008) The observation of the current study revealed that more than two thirds of children (70\%) in study group and nearly half of them $(48.3 \%)$ in control group had right sided inguinal hernia (table 2), which was consistent with a study of 17 children with inguinal hernia there were fourteen hernia $(82 \%)$ were rightsided and three (18\%) left-sided (Wright., et al., 2011).

The result of the current study revealed a highly significant difference between both groups as regard to length of hospital stay $(\mathrm{P}=0.000 * *)$ (table 2), Where Mean \pm SD was $3.2 \pm 0.5 \& 4.9 \pm 2.1$ in the study and control groups respectively, with the average duration of the hospitalization being between 3-5 days in study group. This was consistent with Ravikumar et al., (2013) they reported that most of the patients were treated as in-patients, with the average duration of the hospitalization being between 1-6 days.

The results of the current study revealed a highly significant difference between study and control groups regarding physiological assessment (table 3), where temperature assessment was done in the majority of children in study group compared to only one third of them in control group. While blood pressure monitoring was done in more than half of children in study group compared to none in control group .This was consistent with the study of Castledine (2006)who stated that, measuring and recording patient's vital signs accurately is important as this give an indication of the patient's physiological state.

The results of current study revealed a significant difference between the study and control groups regarding to care of intravenous cannulas (table 4) , where observation of insertion site for absence of infiltration, phlebitis, or inflammation was done in all children $(100 \%)$ in study group compared to nearly two thirds $(65 \%)$ of them in control group, implementing this standards of care was very necessary which was supported by Costello et al., (2010) who reported that excessive transfusion has been associated with both catheter- related blood 
stream infections and surgical site infections in pediatric cardiac patients.

Regarding the comparison between the study and control groups in relation to their wound character it was found that there is no significant difference regarding wound type where almost all children $(100 \%)$ in both groups were with clean surgical wound (table 8 ). More than two thirds $(81.7 \%)$ of children in control group had scrotal swelling compared to only $8.3 \%$ of children in study group after one week of surgery. On the other side there was only $6.7 \%$ of children in control group had scrotal swelling compared to none in study group after one month of surgery. That can be explained in the light of study group who are cared by standard of nursing care that enhance the improvement in their postoperative outcomes.

Also there is no evidence of superficial wound infection in both groups (table 9). This was supported by the study of Robert et al., (2000) that conducted on 92 children with inguinal hernia supported the current study, where they reported that there was no statistically or clinically different between the study and control groups regarding to postoperative wound infection, readmission, and return visits to the Emergency Department (ED) within 72 hours.

Regarding Fever, it was revealed that nearly half of children $(48.3 \%)$ in control group had fever compared to only $13.3 \%$ of children in study group in the $3^{\text {rd }}$ day of surgery (table 9). This was consistent with the studies of (Oeyen $2007 \&$ sinuff et al., 2007) have found that collaborative development of protocols is critical for successful adaptation of protocols and for closing the translational gap in practice. Therefore, our findings support that fever management protocols need to be developed in collaboration with nursing to ensure best-practice is achieved. Furthermore, while protocols were seen as valuable by many participants in the present study, when not applied in a critical manner, they may also be practice-limiting as individual nurses do not think beyond the protocol to what is best for the individual patient.

In the surgical setting, clinical pathways $(\mathrm{CP})$ and standards of care are an excellent means of polling surgical expertise and optimal methods of patient care. By incorporating evidence-based treatment strategies, clinical pathways or standards of care can ensure that medications, laboratory tests and other fundamentals of surgical patient care have been validated for effectiveness, thus eliminating methods of care known to be unnecessary or ineffective (Robert et al., 2000).

The results of current study revealed a significant difference between the study and control groups regarding to management of nutrition for children with inguinal hernia(table 7),Implementing this standards of care was very necessary which was supported by the study of Lawson et al., (2013)who stated that dependent functional status has been associated with higher SSI rates when compared to independent status. Furthermore, hypoalbuminemia is associated with an increased risk of postoperative SSI of any type, and therefore ensuring adequate preoperative nutritional support is essential for optimizing surgical outcomes (Haridas \& Malangoni, 2008).

Finally, it can be concluded that developing and implementing nursing care standard is considered a corner stone in providing efficient and accurate nursing care for the surgical patients in general and patients with inguinal hernia specifically.

\section{Conclusion}

The present study is concluded that, the standard of nursing care improving the postoperative outcomes for children with inguinal hernia as well as it reducing the length time of hospitalization.

\section{Recommendations}

Based on the result of this study, the following recommendations are suggested:

1-Applying of standard of care is an effective method in management of children undergoing inguinal hernia surgery.

2- Educational programs should be provided to increase skills in applying pre and postoperative nursing care standard for children with inguinal hernia.

\section{Future research}

1-This study should be replicated and expanded to include a larger sample, focusing on outcomes with different children populations undergoing a variety of surgical operations in various settings.

2- Similar study should be done to evaluate the effect of implementation of the proposed nursing care standard at different pediatric settings.

\section{References}

1- Abantanga F., Lakhoo K., (2011): Inguinal and femoral hernias and hydroceles, ch .58, in: Paediatric Surgery: A Comprehensive Text For Africa. pp. 358-364.

2- 2-Alam I., Khan M., Jeelani S., Ahmad M., (2013): Clinico-Pathological Study of InguinoScrotal Swelling in Children. Journal of $\mathrm{jk}$ Practitioner;18(3-4):1-3.

3- American Nurses Association (2010): Standards of professional nursing practice .In: Scope and Standards of Nursing Practice, 
Chap(10), $2^{\text {nd }}$ ed., Lippincott Williams\& Wilkins Company, pp.31-39.

4- Brandt M., (2008): Pediatric Hernias. Surgical Clinics journal of North America; 88(1):27-43.

5- Castledine G., (2006): The Importance of Measuring and Recording Vital Signs Correctly. British Journal of Nursing;15 (5):285-285.

6- Costello J., Graham D., Morrow D., Morrow J., Potter-Bynoe G., Sandora T., Pigula F., Laussen P., (2010): Risk Factors for Surgical Site Infection after Cardiac Surgery in Children. The Annals journal of Thoracic Surgery;89:1833-1842.

7- Haridas M., Malangoni M., (2008): Predictive Factors for Surgical Site Infection in General Surgery. Surgery; 144 (4):496-501.

8- Hughes I., Acerini C., (2008): Factors Controlling Testis Descent. European Journal of Endocrinology; 159 (1):75-82.

9- Kareem A., Juma'a K., (2009): Herniotomy in Infants, Children and Adolescents without Disruption of External Ring, World Journal of Laparoscopic Surgery;2(1):13-16

10- Klipfel J., Jacobson T., Havel M., (2010): Radical Nephrectomy with Inferior Vena Cava (IVC) Thrombectomy: Implications for Postoperative Nursing Care. Journal of Urological Nursing; 30 (6):347-352.

11- Lawson E., Hall B., Ko C., (2013): Risk Factors for Superficial vs Deep/Organ-space Surgical Site Infections: Implications for Quality Improvement Initiatives. JAMA Surg; 148 (9):849-858.

12- Marquis B., Huston C., (2010): Leadership roles and management functions in nursing. In: Theory and Application. $5^{\text {th }}$ ed., Philadelphia: Lippincott Williams \&Wilkins. pp.73-90.

13- McKenzie M., Nunn A., Zaller N., Bazazi A., Rich J., (2009): Overcoming obstacles to implementing methadone maintenance therapy for prisoners: implications for policy and practice. Journal of Opioid Management;5:219227.

14- Nagraj S., Sinha S., Grant H., Lakhoo K., Hitchcock R., Johnson P., (2006): The Incidence of Complications Following Primary Inguinal Herniotomy in Babies Weighing $5 \mathrm{~kg}$ or Less. Pediatr SurgInt; 22(6):500-502.

15- Osifo O., Osaigbovo E., (2008): Congenital Hydrocele: Prevalence and Outcome among Male Children who Underwent Neonatal Circumcision in Benin City, Nigeria. Journal of Pediatric Urology; 4(3):178-182.

16- Oeyen S., (2007): Closing the gap between knowledge and behavior: mission impossible? Critical Care Medicine; 35(9):2219-2220.

17- Park M., Park H., (2010): Development of a Nursing Practice Guideline for Pre and PostOperative Care. Health c Inform Res; 16(4): 215-223.

18- Pires M., Pedreira M., Peterlini M., (2013): Safe Pediatric Surgery: Development and Validation of Preoperative Interventions Checklist.Rev. Latino-Am. Enfermagem; 21 (5).

19- Ravikumar V., Rajshankar S., Hareesh R., Kumar S., Nagendra Gowda M., (2013): Clinical Study on the Management of Inguinal Hernias in Children on the General Surgical Practice ,J Clin Diagn Res; 7(1): 144-147.

20- Robert E., Kelly J., Andrea W., Charles J., Donald N., Daniel C., John P., (2000): The Effects of a Pediatric Unilateral Inguinal Hernia Clinical Pathway on Quality and Cost. Journal of Pediatric Surgery; 35(7):1045-1048.

21- Rotter T., Kinsman L., James E., Machotta A., Gothe H., Willis J., (2010): Clinical Pathways: Effects on Professional Practice, Patient Outcomes, Length of Stay and Hospital Costs. Cochrane Database of Systematic Reviews ;Issue 3.

22- Sinuff T., Cook D., (2007): Giacomini M, Heyland D, Dodek P. Facilitating clinician adherence to guidelines in the intensive care unit: a multicenter, qualitative study. Critical Care Medicine; 35(9):2083-2089.

23- Steven L., Joseph M., Roman M., (2011): A critical Review of Premature Infants with Inguinal Hernias: Optimal Timing of Repair, Incarceration Risk, and Postoperative Apnea. Journal of Pediatric Surgery; 46, 217-220.

24- Worled Health Organization (2004): Evidence-led Obstetric Care: Report of a WHO meeting. Geneva: World Health Organization.

25- Wright M., Scollay J., McCabe A., Munro F., (2011): Pediatric femoral hernia. The diagnostic challenge. International Journal of Surgery; 9(6): 472-474. 